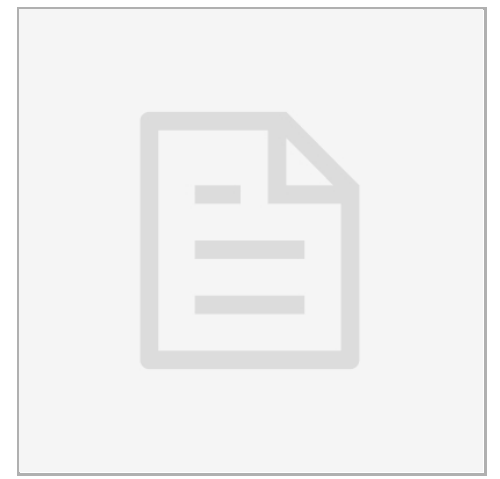

FEB 10, 2020

\section{open $\boldsymbol{\text { Access }}$}

\section{DOI:}

dx.doi.org/10.17504/protocol s.io.bcctiswn

Protocol Citation: Ioannis Bellos, Georgios Daskalakis, Vasilios Pergialiotis 2020. Relationship of vancomycin trough levels with acute kidney injury risk: an exposure-toxicity metaanalysis. protocols.io https://dx.doi.org/10.17504/p rotocols.io.bcctiswn

License: This is an open access protocol distributed under the terms of the Creative Commons Attribution License, which permits unrestricted use, distribution, and reproduction in any medium, provided the original author and source are credited

Protocol status: Working We use this protocol and it's working

Created: Feb 10, 2020

Last Modified: Feb 10, 2020

PROTOCOL integer ID: 32883

\section{(3) Relationship of vancomycin trough levels with acute kidney injury risk: an exposure-toxicity meta-analysis}

\author{
Georgios \\ loannis Bellos ${ }^{1}$, Daskalakis $^{1}, \quad$ Pergialiotis $^{1}$ \\ ${ }^{1}$ National and Kapodistrian University of Athens, Greece
}<smiles>C1CCCCCCCC1</smiles>

Ioannis Bellos

\section{ABSTRACT}

Vancomycin is a tricyclic glycopeptide antimicrobial agent with activity against gram-positive microorganisms, while nephrotoxicity represents one of its main adverse effects. Several risk factors have been identified including vancomycin dose and patients comorbidities, while measurement of trough levels is recommended in order to ensure treatment efficacy and minimize the risk of toxicity. However, the exact relationship of vancomycin trough levels and acute kidney injury incidence remains unclear. The aim of this meta-analysis is to evaluate the association between trough levels and risk of renal impairment, by defining an exposure-toxicity relationship and assessing their accuracy in predicting the development of acute kidney injury.

\title{
1 Review title: Relationship of vancomycin trough levels with acute kidney injury risk: an exposure-
}


toxicity meta-analysis

2 Review question: The meta-analysis aims to clarify whether higher vancomycin trough levels are linked to increased acute kidney injury risk and define an exposure-response relationship between them. Population: Adult hospitalized patients Exposure: Intravenous vancomycin treatment Comparison: High vs. low vancomycin trough levels Outcome: Incidence of acute kidney injury Study type: Randomized controlled trials or observational (prospective or retrospective) studies

3 Searches: The following databases will be systematically searched: MEDLINE, Scopus, ClinicalTrials.gov, CENTRAL, Google Scholar. The reference list of the included studies and previous systematic reviews in the field will be also searched in order to identify potential additional sourced (snowball method). The key-terms that wil be applied would include: "trough, levels, concentration, vancomycin, acute kidney injury, nephrotoxicity, renal failure, renal impairment".

4 Condition or domain being studied: Vancomycin is a tricyclic glycopeptide antimicrobial agent with activity against gram-positive microorganisms, while nephrotoxicity represents one of its main adverse effects, with an incidence ranging from 5-35\%. The pathophysiology of acute kidney injury following vancomycin administration is multifactorial and is considered to be based on mitochondrial dysfunction and increased oxidative stress, leading to tubulointerstitial ischemic damage. Several risk factors have been identified including vancomycin dose and patients comorbidities, while measurement of trough levels is recommended in order to ensure treatment efficacy and minimize the risk of toxicity. However, the exact relationship of vancomycin trough levels and acute kidney injury incidence remains unclear.

5 Participants/population: Inclusion criteria: adult patients, treated with vancomycin in hospital or intensive care unit with available trough levels (initial or maximum) Exclusion criteria: age <18 years, cystic fibrosis, treated outside hospital, use of any form of renal replacement therapy, kidney transplantation

6 Intervention(s), exposure(s): Exposure: intravenous administration of vancomycin and measurement of initial or maximum trough levels

7 Comparator(s)/control: Patients with high trough levels will be compared to those with low trough levels. Definitions of high and low levles are expected to vary among studies and thus a dose-reponse method is planned to be applied.

8 Types of study to be included: Randomized controlled trials and observational (both prospective and retrosepctive) studies will be held eligible. Case-reports, small case-series (including $<10$ 
patients), conference abstracts, posters and animal studies will be excluded. No date restriction will be applied. No language restriction will be applied, except for articles in non-Latin script alphabets.

9 Main outcome(s): Trough levels among patients with and without acute kidney injury (as continuous variable). Inicidence of acute kidney injury in patients with high vs. low trough levels (as binary variable).

10 Timing and effect measures: Both initial and maximum trough levels will be taken into account and analyzed separately. Trough levels will be measured in $\mathrm{mg} / \mathrm{L}$. Acute kidney injury will be defined according to the vancomycin consensus, KDIGO, AKIN or RIFLE guidelines.

11 Data extraction (selection and coding): The meta-analysis will follow the PRISMA guidelines. Selection of studies will be performed in 3 stages. Firstly, all electronic articles articles will be screened by reading their title and abstract. Subsequently, studies that are presumed to be eligible will be retrieved in full-texts. Then, only studies reporting the outcomes of interest and are in accordance with the pre-defined inclusion and exclusion criteria of this meta-analysis will be included. The extracted data will include: year of publication, name of first author, study design, eligibility criteria, measurement of initial or maximum trough levels, indication of vancomycin, clinical setting, use of loading dose, patients' age, renal function, co-administration of nephrotoxins and the outcomes of interest (trough levels and acute kidney injury incidence). Data extraction will be conducted by two authors, while any disagreements will be resolved by their consensus.

12 Risk of bias (quality) assessment: Risk of bias in randomized controlled trials is planned to be evaluated by the Cochrane risk of bias tool, evaluating random sequence generation, blinding, allocation concealment, incomplete outcome and selective reporting. The quality of observational studies will be assessed using the ROBINS-I tool which takes into account the potential bias coming from confounding, selection, classification, deviation from intended intervention, missing data, measurement and reporting of the outcomes. Credibility of evidence will be judged under the GRADE framework. Quality assessment will be performed indepedently by two authors and any disagreements will be resolved by their consensus.

13 Strategy for data synthesis: Meta-analysis will be performed in Review Manager 5.3, Open MetaAnalyst and $\mathrm{R}$ software. Confidence intervals will be set at $95 \%$. Trough levels will be initially analyzed as a continuous varibale among patients with and without acute kidney injury. A random effects model will be applied to provide pooled estimated. Prediction intervals will be also estimated. Subsequently, a dose-response method will be apllied implementing both a linear and non-linear model with restricted cubic splines and the relationship between trough levels and acute kindey risk will be graphically depicted. Linearity will be assessed with the Wald test. A diagnositc accuracy analysis will be also performed using a bivariate model for pre-specified cutoffs (15 and $20 \mathrm{mg} / \mathrm{L}$ ). Sensitivity, specificty and AUC of trough levels for the prediction of acute 
kidney injury will be calculated and a summary ROC curve will be constructed. Publication bias will be assessed by visual inspection of funnel plots, along with the use of statistical tests (regression and rank correlation tests) in homogeneous outcomes including more than 10 studies.

14 Analysis of subgroups or subsets Subgroup analysis: incidence of severe acute kidney injury, use of initial or maximum trough levels Sensitivity analysis: patients in the intensive care unit All analyses will be separately performed in the above subgroups, provided that enough studies $(>5)$ will be available.

15 Key-words: vancomycin trough nephrotoxicity acute kidney injury meta-analysis 